\title{
A Novel Transmit Antenna Selection Algorithms to Improve Energy and Spectral Efficiency
}

\author{
I VeeraraghavaRao, K S Chakradhar, I Durga Prasad, P Raju, V. MalleswaraRao
}

\begin{abstract}
In massive MIMO systems, the selection of optimal transmits antennas remains as a major constraint. As the number of antennas is increased, the power or energy consumption also increases. Selection of optimal transmit antennas is considered as a multi objective problem, where the energy has to be minimizedand the spectral efficiency (bandwidth) has to be increased. In fact, for attaining higher bandwidth, more transmit antennas have to be selected, which leads to increase in power consumption, In this proposal various papers are reviewed for Energy and Spectral Efficiency performance in Massive MIMO Technology through different algorithms and parameter comparisons are made to identify the better algorithms in terms of $E E$ and $S E$ to achieve the higher data transmission rates, BER, mitigating the inter Noise interference.
\end{abstract}

Keywords: Massive MIMO, Antenna Selection, Energy Efficiency, Spectrum Efficiency

\section{INTRODUCTION}

5G is the Major Development in WCN (Wire Less Communication Networks) where the higher data transmission rates are achieved with Massive MIMO by imposing more number of antennas at BS (Base Station). Increasing the number of antennas at BS will increase the power consumption and Channel bandwidth which affect the Energy and Spectrum Efficiencies of system. Consequently the channel Interference [1] also increased, to solve the channel interference problems simultaneous transmission and alignment of channels needed. In view of bandwidth $\mathrm{mm}$ waves [2] play an important role in high frequency configurations. Numerous RF chains are required to achieve high data rates, the RF chains in UP Link and Down Link [3] [9] configuration may affect the EE and SE. Transmit antenna selection (TAS) is also an essential for Massive MIMO [4] [5] in both transmitting and receiving ends. Power consumption at transmitter Circuits [6] and at other relay circuits depends on modulation schemes. The question rises that the Resource allocation and channel characteristics [7] at transmitter optimizes the performance of system. TAS in traditional systems [8] has numerous advantages in perspective of EE Complexity and cost of hardware of the systems [10] affect the RF chains at both transmitter and receiver. This papercontributed the approach of several

Revised Manuscript Received on December 13, 2019.

I. VeeraraghavaRao, Asst.Professor, Dept. of ECE, KL deemed to be University raghva.inumula@gmail.com

K S Chakradhar,Asst. Professor, Dept. of ECE,SrividhyaNiketan College Of Engineringe, Titupati. chakradharec@gmail.com,

T Durga Prasad,durgaprasad.tumula@gitam.edu

P Raju,raju.paladugu@gitam.edu,

Dr. V. Malleswara Rao,malleswararao.veera@gitam.edu.

Department of EECE,GITAM (Deemed to Be University), RUSHIKONDA, VISAKHAPATNAM, 530045 authors for Enhancing Both Energy Efficiency [EE] and spectral [SE] and the trade of between EE and SE, what are the features and challenges in their contributions are well compared, the proposed scheme is review of different algorithms SWIPT, Beam Forming, ZF Precoding, Zero Gradient, PSO, GS based AS, GEN-SM, CF-Cooperation, GA based AS, in all the algorithms GSA performance for both EE and SE found better. This paper organized as follows. Introduction in SEC-I, Related Works and the Reviews, methodologies, Performance Comparison carried in SEC-II, SEC-III and SEC-IV Concludes the paper and References in SEC-V and SEC-VI.

\section{LITERATURE REVIEW}

\section{A. Related Works}

In 2017 H. Zhao[1] Has introduced an algorithm which deals with interference alignment and power splitting architecture for Information Decoding and Energy Harvesting at each receiver by considering the Quality of Service requirements, The raised non convex EE optimization problem solved by designing combination of Power Splitters, Transmitter Beam formers, Transmitter Power and Receiver Filters, it is compared with TZF solution where it able to eliminate the Interference problem only on Transmitter side not on Receiver side and it concluded with better EE optimization for MIMO interference problems. In 2016 J. Jing [2] had established a scheme for Down Link Multi Users to maximize the Energy Efficiency in Massive MIMO applications through millimeter wave technology and it also targets better use of directive gains with analog beam forming algorithm and Energy Efficiency with Digital beam forming algorithms, It also proved that better BER (Bit Error Rate) compared to all traditional Beam forming algorithms when number of users increased.

In 2015 L. Zhao have evaluated Massive MIMO-OFDM with Zero Forcing and Maximum Ratio Combination to enhance the Energy and Spectral Efficiency in Uplink applications, he also proposed a tradeoff between EE and SE when composing the Base Station with number of antennas,

found that the performance of the system performance not enhanced simply by increasing the spatial or frequency resources which also required to improve the rational parameters as number of multiplexing users, different detectors, number of antennas at base station with radiated power. In $2017 \mathrm{Z}$. Liu have investigated a tradeoff between EE and SE using Linear Precoding and TAS 
(Transmit Antenna selection) in Massive MIMO systems, this trade off purely resolved the Complexity about Multi Objective Optimization (MOO) problem and proved that this problem neither Convex or Concave, Optimization of SE and EE done based on transmit antennas and transmit power by considering circuit power consumption and large scale fading.

In 2018 Y. GAO, had introduced a Full-array and Sub-Array switching networks For the Antenna Selection in Massive MIMO channels, he analyzed branch and Bound algorithms for antenna selection, this investigation produced an effective outcomes for Finite Dimensional MIMO.

In 2016 P. Patcharamaneepakorn [6], has introduced a Generalized Modulation technique for $5^{\text {th }}$ generation wireless networks in massive MIMO systems to enhance EE and SE, this proposal very suitable to homogeneous networks and smaller dimensional Antenna Arrays, and investigated a tradeoff between EE and SE, work is very specific to the MF and $\mathrm{ZF}$ Linear receivers.

In 2014 Jing Jiang has suggested an optimization technique in Virtual MIMO systems to enhance the Energy Efficiency, under this article the EE done based on allocation of power to the transmitter and the relays, allocation of bandwidth to the data and cooperation channels, a smaller dimensions 2 X2 had been considered for the MIMO system, EE comparison made between MIMO and MISO, carried out results proved MIMO have better EE when relays provided with extra power.

In 2016 Ngoc Phuc Le had introduced Antenna Selection strategies for MIMO-OFDM systems in the perspective of Energy Efficiency and also made a tradeoff between EE-SE, he proved that The GA based antenna selection provide better EE compared to conventional MIMO systems but due to the complexity of the design and Performance issues it is found that the degradable performances in EE.

\section{B. Review}

Following Table 1 shows the adopted methodology, features and challenges of conventional algorithms designed for Enhancing Energy and Spectral Efficiencies in MIMO and Massive MIMO systems, at first SWIPT SINR-ID suggested in [1] that eliminates interference with improved EE. However due to nonlinear and concave problems affects the system performance. Hybrid Beam Forming using Zero gradient introduced in [2] that mitigate the inter user interference and also minimize the complexity, it offers better candidate solution for EE but it is modeled ad base band processing. $\mathrm{ZF}$ and MRC in [3] that provides excellent tradeoff between EE-SE and the amount of antennas also chosen based on requirement, single cell interference may be the problem. PSO IN [4] was suggested better tradeoff between EE-SE but the Energy Efficiency inter related with SE, EE decreased with SE. GS-AS [5] evaluated to improve the SE lower with lower complexity RF networks in small antenna dimensions, whereas antenna dimensions are increased it gave less power efficiency system performance purely based on MIMO dimensions. GEN-SM in [6] that offers better tradeoff between SE-EE for homogeneous networks, the correlation coefficient may be the factor to discuss in massive antenna arrays. CF- Cooperation [7] evaluated the comparison of EE performance in MIMO and
MISO found to be good EE in MIMO but it required extra power for relays to achieve better EE. GA-AS [8] that suggested a lower complex system for SE-EE tradeoff provide better EE compared to conventional systems, multi user MIMO-OFDMA and larger array antennas are considerable problems.

Table-I: Survey on Existing Massive MIMO Technology Methods for EE and SE Optimization

\begin{tabular}{|c|c|c|c|}
\hline $\begin{array}{l}\text { Author } \\
\text { [citation] }\end{array}$ & $\begin{array}{l}\text { Adopted } \\
\text { methodology }\end{array}$ & Features & Challenges \\
\hline H. Zhao [1] & $\begin{array}{l}\text { The MAX-SINR } \\
\text { ID Algorithm } \\
\text { using SWIPT }\end{array}$ & $\begin{array}{l}\text { Improved EE, } \\
\text { Eliminate } \\
\text { Interference. }\end{array}$ & $\begin{array}{l}\text { Power } \\
\text { Efficiency, } \\
\text { Non Linear } \\
\text { and Concave } \\
\text { Problems }\end{array}$ \\
\hline $\begin{array}{l}\text { J. Jing } \\
{[2]}\end{array}$ & $\begin{array}{l}\text { Hybrid Beam } \\
\text { forming using } \\
\text { ZERO-gradient }\end{array}$ & $\begin{array}{l}\text { Better EE, } \\
\text { mitigate Inter } \\
\text { user interference. }\end{array}$ & $\begin{array}{l}\text { Base band } \\
\text { Processing, } \\
\text { less Power } \\
\text { efficiency } \\
\end{array}$ \\
\hline L. Zhao [3] & $\begin{array}{l}\text { ZF Detection, } \\
\text { Maximum Ratio } \\
\text { combination }\end{array}$ & $\begin{array}{l}\text { Trade of between } \\
\text { EE and SE, } \\
\text { Provides the } \\
\text { Desired amount } \\
\text { of antennas } \\
\text { chosen. }\end{array}$ & $\begin{array}{l}\text { In single cell } \\
\text { condition } \\
\text { inter cell } \\
\text { interference } \\
\text { problem. }\end{array}$ \\
\hline $\begin{array}{l}\text { Z. Liu } \\
\text { [4] }\end{array}$ & $\begin{array}{l}\text { Particle Swarm } \\
\text { optimization } \\
\text { (NBI,WS) }\end{array}$ & $\begin{array}{l}\text { Excellent } \\
\text { trade-off } \\
\text { Between EE,SE }\end{array}$ & $\begin{array}{l}\text { EE } \\
\text { Decreases } \\
\text { with } \\
\text { Decrease in } \\
\text { SE }\end{array}$ \\
\hline $\begin{array}{l}\text { Y. Gao } \\
{[5]}\end{array}$ & $\begin{array}{l}\text { Greedy search } \\
\text { Based Antenna } \\
\text { Selection }\end{array}$ & $\begin{array}{l}\text { Minimized } \\
\text { Complexity, } \\
\text { Better Spectrum } \\
\text { Efficiency }\end{array}$ & $\begin{array}{l}\text { RF Fully } \\
\text { switching } \\
\text { networks are } \\
\text { less power } \\
\text { Efficient } \\
\text { with Larger } \\
\text { MIMO } \\
\end{array}$ \\
\hline $\begin{array}{l}\text { PiyaPatchara } \\
\text { maneepakorn } \\
{[6],}\end{array}$ & $\begin{array}{l}\text { Generalized } \\
\text { Spatial } \\
\text { Modulation }\end{array}$ & $\begin{array}{l}\text { Improved EE, } \\
\text { Moderate SE }\end{array}$ & $\begin{array}{l}\text { Gen-SM for } \\
\text { Hetero } \\
\text { Genius N/W, } \\
\text { CC for } \\
\text { massive } \\
\text { Antenna } \\
\text { Arrays } \\
\end{array}$ \\
\hline Jing Jiang [7] & $\begin{array}{l}\text { Compress and } \\
\text { Forward } \\
\text { Cooperation }\end{array}$ & $\begin{array}{l}\text { Enhance EE for } \\
\text { MIMO over } \\
\text { MISO }\end{array}$ & $\begin{array}{l}\text { Shared Band } \\
\text { Cooperation } \\
\text { Channels, } \\
\text { Extra Power } \\
\text { for Relays }\end{array}$ \\
\hline $\begin{array}{l}\text { Ngoc Phuc } \\
\text { Le [8] }\end{array}$ & $\begin{array}{l}\text { GA based } \\
\text { Antenna } \\
\text { Selection }\end{array}$ & $\begin{array}{l}\text { Better EE } \\
\text { compared to } \\
\text { conventional } \\
\text { Systems, lower } \\
\text { Complexity }\end{array}$ & $\begin{array}{l}\text { Multi User } \\
\text { MIMO-OFD } \\
\text { MA, Larger } \\
\text { Antenna } \\
\text { Arrays }\end{array}$ \\
\hline
\end{tabular}

\section{PRECODING METHODOLOGY}

To maintain the variations in the stream of information's we mostly used Precoding methodology, treated as $\mathrm{S}$ be the amount of information and Channel as $\mathrm{H}$, the additive Gaussian noise can be treated as $\mathrm{N}$ after the amount of information received at receiver is given in Equation (1)

$$
R=(S) H+N
$$


From the proposed equation (1), receiver must have the information and clarity of $\mathrm{H}$ and $\mathrm{N}$ also this makes the raise in design complexity of receiver, in generally max of practical receivers are simple and easier. So that most of the Transmitters use the Precoding methods to transmit the data as $\boldsymbol{S} / \boldsymbol{H}_{\boldsymbol{E S T}}$ then the received information at receiver part is equation (2)

$$
\mathrm{R}=\left(\frac{\mathrm{S}}{\mathrm{H}_{\mathrm{EST}}}\right) \mathrm{H}+\mathrm{N}
$$

Assume correct prediction by $\mathbf{H}_{\text {EST }}=\mathbf{H}$, then the signal of receiver becomes $\boldsymbol{R}=\boldsymbol{S}+\boldsymbol{N}$, it is fact that the method of Precoding is eliminate or minimize the error at receiver part. To attain better EE and SE performance in Massive MIMO Technology $\mathrm{ZF}$ (Zero Force) and MF (Matched filtering) methods will use.

\section{RESULT \& PERFORMANCE COMPARISION}

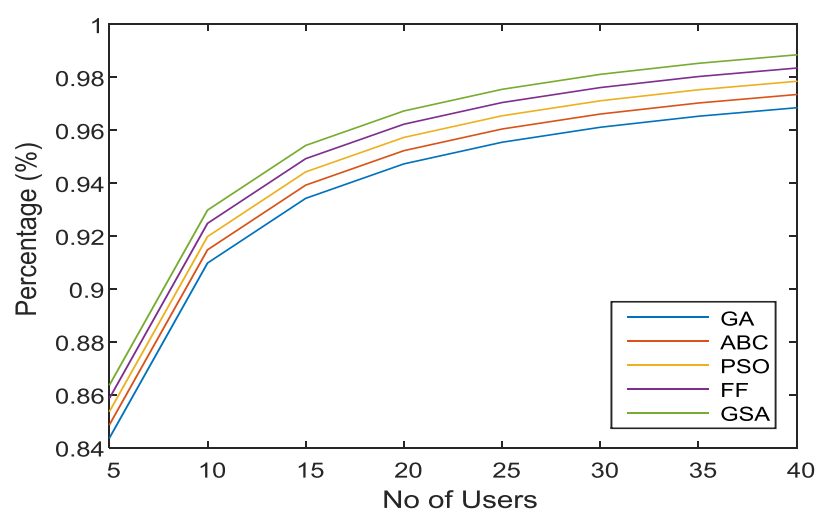

Fig 1. Zero Force Precoding

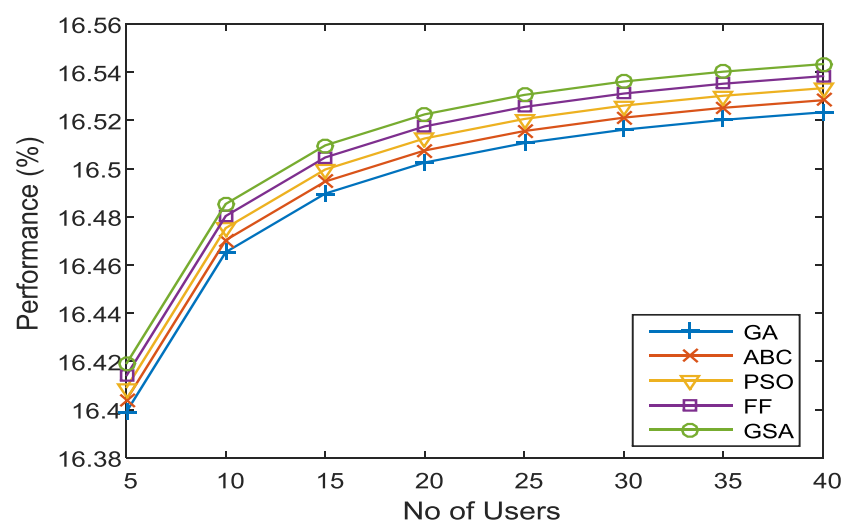

Fig 2. Matched Filter Precoding

From the Figures 1, 2 a comparison made with different algorithms which are available for Precoding methods to Maximize EE and SE, among GSA will be the better in ZF and MF in performance criteria.

\section{EE an SE with respect to the Number of Antennas}

The EE is defined as the number of bits transferred per Joule of energy.

$$
\begin{aligned}
& E E=\frac{C}{P_{\text {sum }}} \\
& f_{E E}\left(N_{t}, P_{t}\right)=\frac{B \cdot K \cdot \log _{2}\left(1+\frac{P_{t}\left(N_{t}-K\right)}{K \bar{\alpha}-\sigma^{2}}\right)}{\frac{B \cdot P_{t}}{n}+N_{t} P_{B C}+P_{\text {etc }}}
\end{aligned}
$$

The Spectral Efficiency is defined as the sum-rate per unit of bandwidth.

$$
\mathrm{f}_{\mathrm{SE}}\left(\mathrm{N}_{\mathrm{t}}, \mathrm{P}_{\mathrm{t}}\right)=\mathrm{K} \log _{2}\left(1+\frac{\mathrm{P}_{\mathrm{t}}\left(\mathrm{N}_{\mathrm{t}}-\mathrm{K}\right)}{\mathrm{K} \bar{\alpha}_{-1} \sigma^{2}}\right)
$$

The Energy Efficiency and Spectral Efficiency is related as

$$
\mathrm{f}_{\mathrm{EE}}=\frac{\mathrm{B} \cdot \mathrm{f}_{\mathrm{SE}}}{\frac{\mathrm{B}\left(2^{\frac{\mathrm{f}_{\mathrm{SE}}}{\mathrm{K}}-1}\right) \cdot \frac{\mathrm{K} \bar{\alpha}_{-1} \sigma^{2}}{N_{t}-\mathrm{K}}}{\eta}+\mathrm{N}_{\mathrm{t}} \mathrm{P}_{\mathrm{BC}}+\mathrm{P}_{\text {etc }}}
$$

\section{CONCLUSION}

From the execution outcomes, In Massive MIMO Technology many of the algorithms are trying to achieve antenna optimization and Energy and Capacity optimization but when comparison made only GSA will be the leading partner in antenna optimization and Capacity, Energy Optimization, This result concludes that GSA will be suitable for Massive MIMO applications.

\section{REFERENCES}

1. Huimin Zhao, Zujun Liu, Yongjun Sun, "Energy efficiency optimization for SWIPT in K-user MIMO interference channels" ,Physical Communication, vol. 27, pp. 197-202, April 2018.

2. Jiang Jing, Cheng Xiaoxue, Xie Yongbin, "Energy-efficiency based downlink multi-user hybrid beamforming for millimeter wave massive MIMO system", The Journal of China Universities of Posts and Telecommunications, vol. 23, no. 4, pp. 53-62, August 2016.

3. Long Zhao, Ke Li, Kan Zheng, " An Analysis of the Trade Off

4. Between The Energy \& Spectrum Effeciences in An Uplink Massive MIMO-OFDM System", IEEE TRANSACTIONS ON CIRCUITS AND SYSTEMS, VOL. 62, NO. 3, MARCH 2015.

5. Z. Liu, W. Du and D. Sun, "Energy and Spectral Efficiency Tradeoff for Massive MIMO Systems With Transmit Antenna Selection," IEEE Transactions on Vehicular Technology, vol. 66, no. 5, pp. 4453-4457, May 2017

6. Y. Gao, H. Vinck and T. Kaiser, "Massive MIMO Antenna Selection Switching Architectures, Capacity Bounds, and Optimal Antenna Selection Algorithms," IEEE Transactions on Signal Processing, vol 66, no. 5, pp. 1346-1360, March1, 12018.

7. Piya Patcharamaneepakorn, ShangbinWu, Cheng-XiangWang, "Spectral, Energy, and Economic Efficiency of 5G Multicell Massive MIMO Systems With Generalized Spatial Modulation” IEEE TRANSACTIONS ON VEHICULAR TECHNOLOGY, VOL. 65 , NO. 12, DECEMBER 2016

8. Jing Jiang, Mehrdad Dianati, Muhammad Ali Imran, Rahim Tafazolli, Shunqing Zhang, "Energy-Efficiency Analysis and Optimizatio for Virtual-MIMO Systems", IEEE TRANSACTIONS ON VEHICULAR TECHNOLOGY, VOL. 63, NO. 5, JUNE 2014.

9. Ngoc Phuc Le, Farzad Safaei, Le Chung Tran, “ Antenna Selection Strategies for MIMO-OFDM Wireless Systems: An Energy Efficiency Perspective", IEEE TRANSACTIONS ON VEHICULAR TECHNOLOGY, VOL. 65, NO. 4, APRIL 2016

10. Maryam Olyaee, Mohsen Eslami, Javad Haghighat, “EE joint antenna and user selection algorithm for multi-user massive MIMO downlink", IET Commun., 2018, Vol. 12 Iss. 3, pp. 255-260.

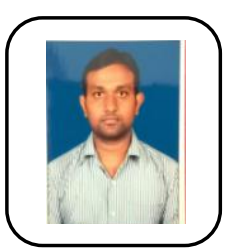

\section{AUTHORS PROFILE}

Inumula Veeraraghava Rao M.Tech, (PhD) Pursuing $\mathrm{PhD}$ in GITAM(Deemed to be University) having keen interest in Wireless Communication for maximizing EE and SE in 5G applications. 


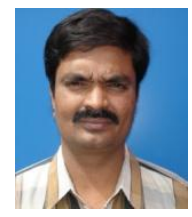

K.S.Chakradhar,completed M.E. in the stream of Electronics \& Control Engineering from Sathyabama Deemed University, Chennai in the year of 2005 and pursuing Ph.D at GITAM university, Visakhapatnam. Currently, working as Associate Professor in SreeVidyanikethan Engineering College (A),Tirupathi, India. Micro strip Patch antennas and VLSI are the interest areas.

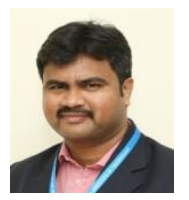

T.Durga Prasad, working as Assistant Professor in GITAM .Areas of Interest are Antennas, Microwave engineering.

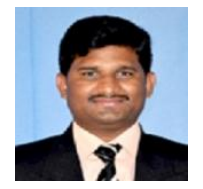

Raju.P, working as Assistant Professor in GITAM .Areas of Interest are Digital image processing,Signalprocessing,Biomedical signal processing

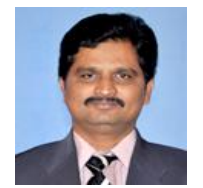

Dr. V. Malleswara Rao, working as Professor in GITAM (Deemed to be University) from past 30 years. He has been published more than 75 articles in national and international Journals. 